\title{
Valor da ressonância magnética no diagnóstico antenatal do acretismo placentário
}

\author{
Value of magnetic resonance imaging in prenatal diagnosis of placental accretism
}

Viviane Vieira Franciscoํㅗ , Suzan Menasce Goldman², Juliano Faria ${ }^{3}$, Jacob Szejnfeld ${ }^{4}$

\section{RESUMO}

Objetivos: estabelecer os principais sinais de acretismo placentário na ressonância magnética (RM) em gestantes com suspeita clínica e avaliar a utilidade do método. Métodos: estudo prospectivo, transversal em 15 pacientes com suspeita de acretismo placentário. O período compreendido foi de março de 2003 a fevereiro de 2006. A idade gestacional variou de 20 a 31 semanas. Todas as pacientes realizaram RM com estudo dirigido para placenta e haviam sido submetidas à ultra-sonografia (US) prévia ao exame. Todas as peças foram encaminhadas para estudo anátomo-patológico (AP). Os exames foram realizados nos equipamentos Magnetom Impact e Sonata Maestro Class Siemens ${ }^{\circledR}$, adquiridas as seqüências HASTE, TURBO FISP, nos planos axial, sagital e coronal e gradiente echo $\left(\mathrm{GE}^{\circledR}\right)$ pré- e pós- contraste dinâmico no melhor plano de aquisição. A análise das imagens foi realizada por dois radiologistas em consenso. Resultados: a idade gestacional média das pacientes foi de 24,3 semanas. Foram estudadas sete placentas prévias centro-totais (47\%), seis placentas corporais anteriores $(40 \%)$ e duas placentas corporais posteriores (13\%). A US foi positiva em $80 \%$ dos casos e a RM em 53\% dos casos. No entanto, a US apresentou concordância fraca com o AP pelo teste de kappa (11\%), com sensibilidade de 75\%, especificidade de $14 \%$, valor preditivo positivo (VPP) de 50\% e valor preditivo negativo (VPN) de 33\%. Já a RM teve concordância excelente com o AP $(0,86)$, com sensibilidade de $100 \%$, especificidade de $86 \%$, VPP de $89 \%$ e VPN de $100 \%$. Conclusões: a RM é útil na identificação do acretismo placentário. Os principais sinais na RM do acretismo placentário são: o hipersinal transmural, a descontinuidade da parede miometrial nas seqüências rápidas e a identificação dos vasos invadindo o miométrio nas seqüências dinâmicas.

PALAVRAS-CHAVE: Placenta acreta/diagnóstico; Imagem por ressonância magnética

\section{ABSTRACT}

Purpose: to establish the main signs of placental accretism in magnetic resonance imaging (MRI) in patients with clinical suspicion and to estimate the benefit of this method. Methods: prospective transversal study with 15 patients suspected of placental accretism, referred between March 2003 and February 2006. Gestational age varied from 20 to 31 weeks. All patients underwent MRI to study the placenta and had previously done an ultrasonography. Material was sent to histological study. MRI was done on Magnetom Impact and Sonata Maestro Class Siemens ${ }^{\circledR}$, with acquired sequences HASTE, TURBO SPIN in axial, sagittal, coronal planes and echo gradient $\left(\mathrm{GE}^{\circledR}\right)$, pre- and post-dynamic contrast in the best plan for acquisition. Images were analyzed by a team of two radiologists. Results: mean gestational age was 24.3 weeks. We studied seven placenta previa (47\%), six anterior placentas (40\%) and two posterior placentas (13\%). Ultrasonography was positive in $80 \%$ of the palcentas and MRI in 53\%. However, echography had a low concordance with anatomic pathological studies by Kappa test (11\%), revealing $75 \%$ of sensitivity, $14 \%$ of specificity, $50 \%$ as positive predictive value (PPV) and $33 \%$ as negative predictive value (NPV). MRI had an excellent concordance with anatomic pathological studies (0.86), showing $100 \%$ of sensitivity, $86 \%$ of specificity, $89 \%$ as PPV and $100 \%$ as NPV. Conclusions: MRI is useful for placental accretism diagnosis. The principal findings are transmural hyper-signal, the loss of continuity in myometrial wall in fast sequences and the identification of vessels invading myometrial layer in dynamic sequences.

KEYWORDS: Placenta accreta/diagnosis; Magnetic resonance imaging

1 Pós-graduanda do Departamento de Diagnóstico por Imagem da Universidade Federal de São Paulo - UNIFESP - São Paulo (SP), Brasil.

2 Professora do Departamento de Diagnóstico por Imagem da Universidade Federal de São Paulo - UNIFESP - São Paulo (SP), Brasil.

3 Pós-graduando do Departamento de Diagnóstico por Imagem da Universidade Federal de São Paulo - UNIFESP - São Paulo (SP), Brasil.

4 Livre-Docente do Departamento de Diagnóstico por Imagem da Universidade Federal de São Paulo - UNIFESP - São Paulo (SP), Brasil. Correspondência: Viviane Vieira Francisco

Rua Pedro Morganti, 76 - Vila Mariana - 04020-070 - São Paulo - SP - Fone: (11) 5573-4326 - Fax: (11) 3288-6040 - e-mail: vieira_francisco@uol.com.br Recebido em: 7/8/2006 Aceito com modificaçōes em: 4/12/2006

Rev Bras Ginecol Obstet. 2006; 28(12): 700-7. 


\section{Introdução}

O acretismo placentário consiste na aderência anormal da placenta na parede uterina. A base histopatológica consiste na ausência ou na desordem da decídua basal, que é a camada mais profunda do endométrio. A aderência placentária anormal incluindo a placenta acreta, increta ou percreta é uma causa freqüente de hemorragia pós-natal ${ }^{1}$. A invasão do vilo corial no miométrio eleva o risco de sangramento, aumentando as chances de transfusões sanguíneas ou até de histerectomia, o que repercurte diretamente na elevação da morbimortalidade ${ }^{1}$.

A prevalência do acretismo aumentou significativamente nos últimos 50 anos, encontrando-se nos trabalhos recentes de 1:540 até 1:93000 partos. A placenta prévia é a causa mais freqüente do acretismo placentário. $\mathrm{O}$ aumento na freqüência do acretismo correlaciona-se ao aumento do número de cesáreas, multiparidade, miomectomias e embolização de miomas ${ }^{1}$.

A extensão da invasão placentária, muitas vezes, não é conhecida até o trabalho de parto. Isso ocorre devido à falta de definição na literatura do melhor método de diagnóstico pré-natal do acretismo placentário, incluindo os sinais radiológicos e marcadores sanguíneos maternos definitivos, e da falta de preparo dos profissionais em pesquisar essa co-morbidade.

A adequada detecção do acretismo placentário e da extensão da invasão miometrial permitiria um adequado planejamento da via de parto, do risco operatório e das medidas de segurança nessas condições. Isso resultaria na redução da morbidade e em uma abordagem multidisciplinar de uma situação potencialmente dramática.

A ultra-sonografia (US), o power doppler e a ressonância magnética (RM) têm sido utilizados como modalidades no diagnóstico do acretismo placentário, porém a US ainda é a modalidade mais acessive $1^{1-3}$

Existem critérios ultra-sonográficos estabelecidos para o diagnóstico da placenta acreta, os quais têm sido utilizados com sucesso relativo. Entretanto, ainda não existem critérios para a $\mathrm{RM}^{2,4}$.

A RM, nos últimos dez anos, se destaca como método de diagnóstico antenatal de malformações complexas e de patologias maternas na gestação, devido a não exposição à radiação ionizante e à boa resolução diagnóstica ${ }^{5}$.

Já existem evidências na literatura de que a RM tem um papel importante no auxílio da detecção e complementa a avaliação da extensão do acretismo e do percretismo placentário; no entanto, estes estudos são limitados, pois as análises são retrospectivas e há grande variabilidade técnica ${ }^{4,5}$.
Neste trabalho, apresentamos os principais sinais na RM que auxiliam o diagnóstico do acretismo placentário, demonstramos que a fase com contraste auxilia a avaliação do acretismo, definimos os principais sinais sugestivos deste diagnóstico e comparamos da sensibilidade da RM e a da US.

\section{Métodos}

Foi realizado um estudo prospectivo transversal no Departamento de Diagnóstico por Imagem (DDI) da Universidade Federal de São Paulo (UNIFESP) de 15 pacientes gestantes encaminhadas pela Disciplina de Obstetrícia, com suspeita clínica de acretismo placentário. O período compreendido foi de março de 2003 até fevereiro de 2006. A idade gestacional variou de 20 a 31 semanas.

Todas as pacientes foram encaminhadas para realizar o exame de RM com protocolo dirigido para avaliação da placenta, após exame ultra-sonográfico prévio. A indicação dos exames foi baseada em manifestações clínicas e/ou ultra-sonográficas que sugeriam o diagnóstico de acretismo placentário. Os estudos foram indicados pela equipe de obstetrícia da Disciplina de Obstetricia da UNIFESP. As pacientes foram instruídas e assinaram termo de consentimento. O trabalho foi autorizado pelo Comitê de Ética e Pesquisa da UNIFESP.

Os critérios clínicos para suspeita de acretismo placentário foram: multiparidade, cesáreas (mais que duas), placenta prévia, placentas corporais anteriores, miomectomias e manipulações cirúrgicas prévias do útero. As gestantes incluídas neste estudo apresentavam idade gestacional acima de 16 semanas, suspeita clínica de acretismo placentário, US na época da solicitação, RM com protocolo dirigido e estudo anátomo-patológico (AP) da placenta.

Os critérios ultra-sonográficos indicativos de suspeita de acretismo placentário foram os preconizados na literatura, destacando-se: o adelgaçamento da zona hipoecóica retroplacentária, lacunas vasculares irregulares, irregularidade na espessura do miométrio basal e presença de vasos tortuosos invadindo o miométrio ${ }^{2,3,6,7}$. Os exames de US foram realizados na disciplina de Obstetrícia, Setor de Medicina Fetal da UNIFESP, por médicos especialistas em US obstétrico e residentes supervisionados pelos especialistas. O equipamento de ultra-som utilizado foi o Logic $400 G E^{\mathbb{B}}$. Os exames de RM foram realizados nos aparelhos Magnetom Impact (1,0 T) e Sonata Maestro Class 1,5 T (Siemens MC). As imagens foram realizadas nas pacientes com bobina de corpo com 12 canais. As seqüências realizadas foram: TSE (coronal, sagital, axial), HASTE TSE nos três planos, TURBO FISP 
nos três planos, seqüências gradiente echo (GE) ponderadas em T1, pré- e pós- contraste no plano de melhor caracterização da placenta. Os parâmetros técnicos das seqüências estão demonstrados

Tabela 1 - Parâmetros técnicos das seqüências estudadas, relacionando as seqüências de ressonância magnética com os parâmetros técnicos da aquisição de imagem no aparelho.

\begin{tabular}{lcccc}
\hline $\begin{array}{l}\text { Parâmetros } \\
\text { técnicos }\end{array}$ & TSE & HASTE & TURBO FISP & SEQ GD \\
\hline TR & 5 & 1000 & 1000 & 5 \\
TE & 2,5 & 121 & 121 & 2,5 \\
FOV $(\mathrm{mm})$ & 330 & 330 & 330 & 330 \\
Matriz & 360 & 360 & 360 & 360 \\
Flip Angle & 70 & 70 & 70 & 70 \\
Espessura (mm) & 4 & 4 & 4 & 4 \\
Gap & 8,1 & 8,1 & 8,1 & 8,1 \\
\hline
\end{tabular}

TR: tempo de repetição; TE: tempo de eco; FOV: field of view (parâmetro que permite dar aumento de resolução de imagem da área estudada); Flip angle: ângulo de aquisição da seqüência; espessura: espessura de corte da seqüência; Gap: intervalo entre cortes.

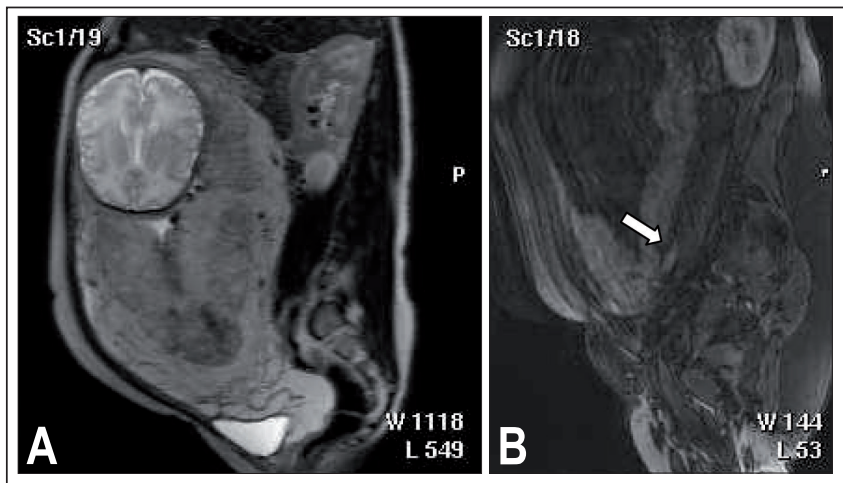

Figura 1 - Gestante 26 semanas, com placenta de inserção baixa e diagnóstico de acretismo pós-natal avaliada na seqüência TURBO FISP (A), bom contraste tecidual, sem a caracterização do acretismo, e na seqüência pós contraste $(B)$ com área de realce no miométrio que correspondeu ao acretismo posterior.

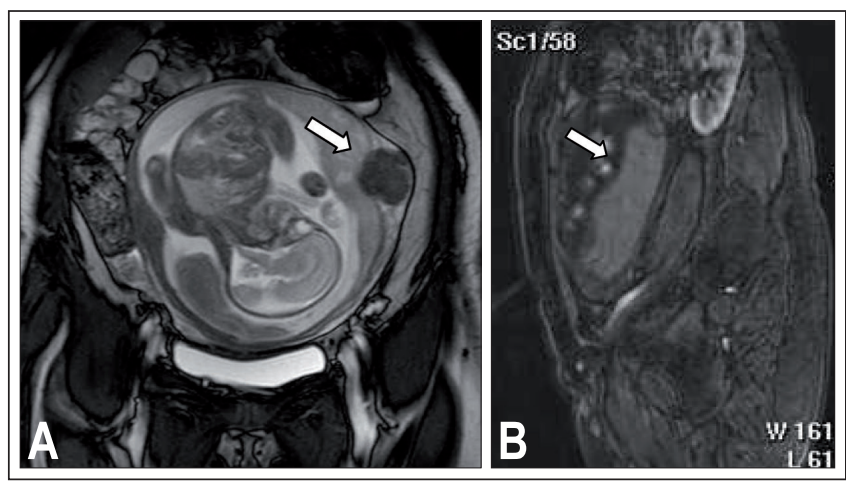

Figura 2 - Gestante de 23 semanas com mioma uterino e suspeita de acretismo. Seqüência TURBO FISP (A), no corte coronal, placenta corporal anterior, lateral esquerda e mioma intramural medindo aproximadamente $4 \mathrm{~cm}$ e difícil definição do acretismo, a seqüência dinâmica (B) caracterizou satisfatoriamente acretismo. na Tabela 1. O contraste utilizado foi o gadolíneo (Gd-DTPA). A dose preconizada de gadolíneo foi de: $0,2 \mathrm{~mL} / \mathrm{kg}$. Os parâmetros técnicos estão demonstrados na Tabela 1.

A análise das imagens foi realizada por dois radiologistas experientes em $\mathrm{RM}$ de abdome em consenso (SMG e JS). Os parâmetros analisados nos exames de RM foram: a localização da placenta, a espessura da parede miometrial, a visualização da invasão transmural da placenta, a invasão de vasos no miométrio na fase mais precoce de aquisição e o envolvimento de órgãos adjacentes (Figuras $1,2,3$ e 4). Os critérios indicativos da presença de acretismo utilizados pelos observadores foram: a espessura miometrial fina, o hipersinal da extensão transmural placentária nas seqüências TURBO FISP e HASTE e o contraste de vasos invadindo o miométrio nas seqüências dinâmicas.

As placentas foram encaminhadas para estudo anátomo-patológico no Departamento de Patologia da UNIFESP; o diagnóstico de positividade para o acretismo foi a presença de tecido miometrial aderido à placenta, a não distinção adequada entre miométrio e placenta, além da perda da membrana basal. Não foi possivel estabelecer o acretismo parcial ou total, de acordo com a profundidade miometrial, pois o útero não foi retirado em todos os casos. Em alguns casos, foi realizada a histerectomia e o útero foi enviado junto com a placenta para a análise anátomo-patológica.

Foi realizada a comparação entre os achados ultra-sonográficos e os da ressonância magnética com o estudo anátomo-patológico. A análise estatística foi realizada com o teste da concordância de kappa e o teste de Fisher para a avaliação da concordância entre US e RM, RM e AP, US e AP.

O índice kappa foi utilizado para avaliar a concordância entre US e RM, e entre estes métodos e o resultado anátomo-patológico.

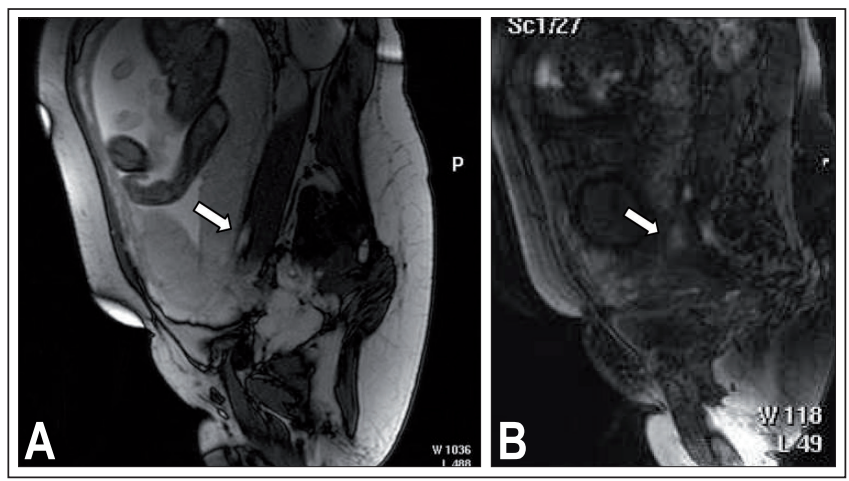

Figura 3 - Gestante de 24 semanas com inserção baixa de placenta e suspeita de acretismo. Seqüência HASTE (A) no corte sagital, placenta prévia com hipersinal transmural no colo, parede posterior, e na seqüência dinâmica (B) vasos invadindo o miométrio. 


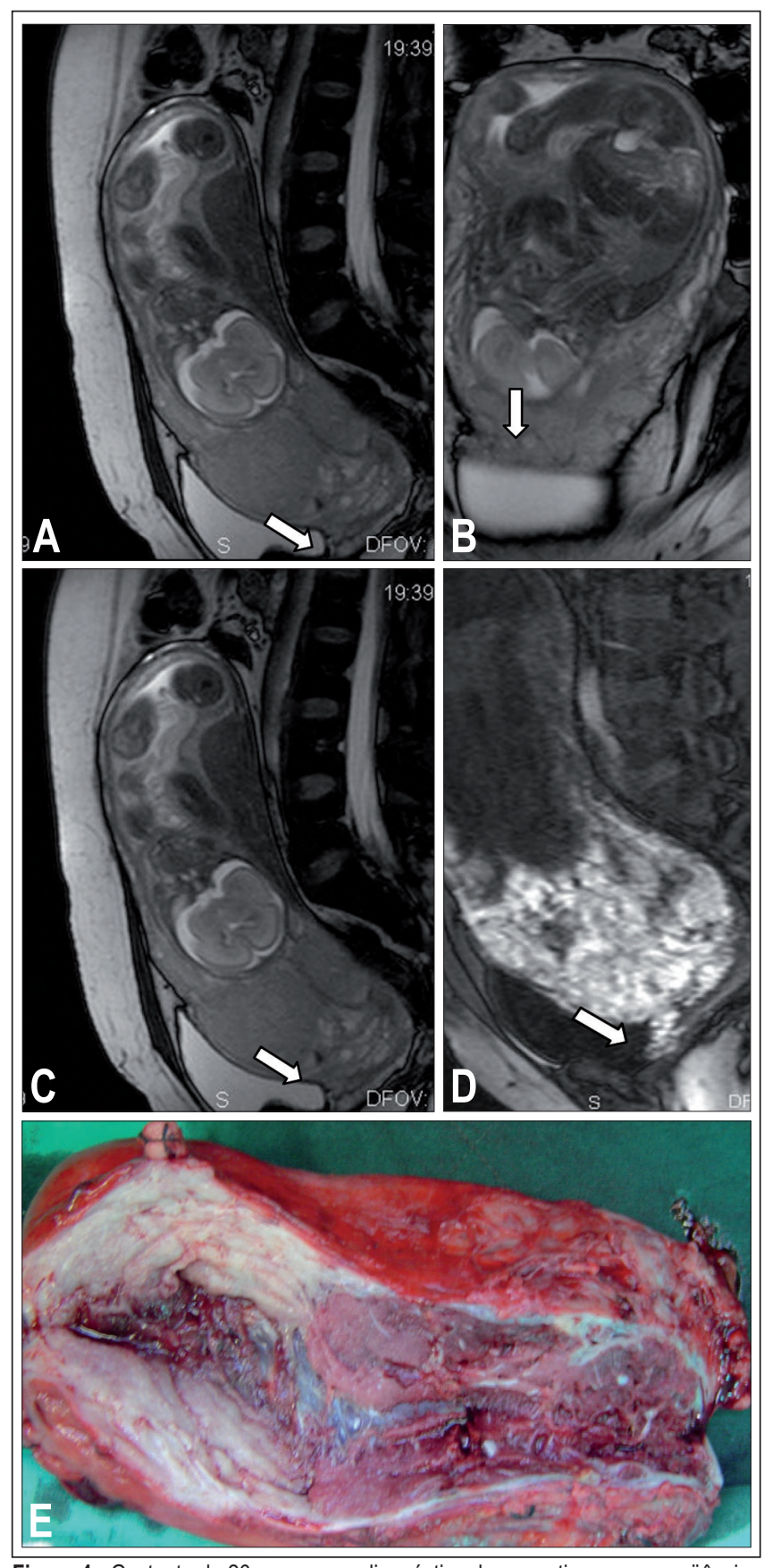

Figura 4 - Gestante de 26 semanas e diagnóstico de percretismo, nas seqüências TURBO FISP (A,B,C); nos três planos evidencia-se hipersinal transmural da placenta, afilamento da parede miometrial e nas seqüências dinâmicas (D) o realce envolvendo a região do trígono vesical $(\mathrm{E})$ peça de histerectomia com a placenta dentro.

\section{Resultados}

A idade gestacional das pacientes variou de 20 a 31 semanas. A mediana foi de 24 semanas (mínimo de 20 e máximo de 31 semanas) e a média representou 24,3 semanas (desvio padrão: 3,4).

A localização placentária em nossa amostra foi: seis placentas corporais anteriores (40\%), sete placentas prévias centro-totais (47\%) e duas placentas corporais posteriores (13\%).

Quinze pacientes com suspeita clínica de acretismo foram estudadas. Neste grupo, 12 (80\%) apresentaram US positiva e nove (53\%), RM positiva para o diagnóstico de acretismo placentário. Do grupo de US positivas, apenas seis tinham acretismo placentário; do grupo de RM positivas, oito tinham acretismo; assim, o diagnóstico de acretismo placentário foi confirmado com o estudo anátomopatológico em oito casos (Tabelas 2, 3 e 4).

Entre os três casos negativos para o acretismo na US, em apenas um houve confirmação da negatividade (Tabela 3). Nos seis casos em que a RM descartou o acretismo, o estudo da peça cirúrgica também foi negativo (Tabela 4).

A avaliação pela ultra-sonografia indicou sensibilidade de $75 \%$, especificidade de $14 \%$, valor preditivo positivo (VPP) de $50 \%$ e valor preditivo negativo (VPN) de 33\% para o diagnóstico de placenta acreta (Tabela 3). A RM neste grupo de pacientes indicou sensibilidade de $100 \%$, especificidade de $86 \%$, VPP de $89 \%$ e VPN de $100 \%$ (Tabela 4). A concordância do estudo US e da RM com o resultado anátomo-patológico foi de $11 \% \mathrm{e}$ $86 \%$, respectivamente.

Houve concordância pobre entre US e AP, mostrando kappa 0,11. A concordância da RM com o AP indicou índice de kappa de 0,86, ou seja, concordância quase perfeita. Dos 15 casos, apenas seis tiveram o diagnóstico de acretismo pelo estudo anátomo-patológico. Dos oito casos considerados positivos para o acretismo, três pacientes foram submetidas a histerectomia e cinco tiveram que receber transfusões de sangue e derivados. Nas pacientes em que o útero não foi retirado, o diagnóstico foi feito com o estudo da placenta. Quando o diagnóstico de acretismo foi estabelecido pela RM, a evolução do trabalho de parto foi modificada na nossa amostra.

Tivemos um caso de percretismo com invasão vesical. Nesta paciente, a gravidez foi interrompida com 26 semanas e quatro dias e a equipe médica consistiu de dois urologistas, ginecologistas oncológicos, obstetras, neonatologistas e anestesistas, pois havia a expectativa de cirurgia de grandes proporções. Isto se confirmou, já que a paciente foi submetida a cistectomia parcial, histerectomia total, com reconstrução imediata e cateterização dos ureteres. A paciente e o feto evoluíram satisfatoriamente. Não houve nenhum caso de complicação pelo contraste gadolinio. Na literatura, os efeitos colaterais deste contraste ainda não estão bem estabelecidos. As gestantes se recuperaram bem do ponto de vista hemodinâmico.

Em resumo, os sinais de acretismo encontrados foram o hipersinal e a descontinuidade da 
Tabela 2 - Características dos pacientes e diagnósticos respectivos realizados pela utra-sonografia e pela ressonância magnética.

\begin{tabular}{|c|c|c|c|c|c|c|}
\hline Paciente & Idade gestacional & Placenta & US & RM & AP & Evolução \\
\hline 1 & 24 semanas & PCA & + & - & - & normal \\
\hline 2 & 22 semanas & PCP & + & + & + & histerectomia \\
\hline 3 & 26 semanas & PP CT & + & - & - & normal \\
\hline 4 & 23 semanas & PCA & + & - & - & normal \\
\hline 5 & 20 semanas & PCP M+ & + & + & + & sangue (Tx) \\
\hline 6 & 21 semanas & PP CT & + & + & + & histerectomia \\
\hline 7 & 20 semanas & PP CT & + & - & - & normal \\
\hline 8 & 21 semanas & PP CT & + & + & + & sangue (Tx) \\
\hline 9 & 23 semanas & PCA & - & + & + & sangue (Tx) \\
\hline 10 & 26 semanas & PP CT & - & + & + & HTA + CTS \\
\hline 11 & 24 semanas & PP CT & + & + & + & sangue (Tx) \\
\hline 12 & 26 semanas & PCAM & + & + & + & sangue (Tx) \\
\hline 13 & 28 semanas & PCAM & + & - & - & normal \\
\hline 14 & 31 semanas & PP CT & + & + & - & normal \\
\hline 15 & 30 semanas & PCA & - & - & - & normal \\
\hline
\end{tabular}

US = ultra-sonografia; RM = ressonância magnética; $A P$ = anátomo patológico; $P C A$ = parede corporal anterior; $P C P=$ parede corporal posterior; $P P C T$ = placenta prévia centro total; PCA M = parede corporal anterior com mioma; HTA = histerectomia; CST = cistectomia; (Tx) = transfusão de sangue e derivados.

Tabela 3 - Concordância entre o ultra-som (US) e o resultado anátomo-patológico (AP).

\begin{tabular}{lccc}
\hline Teste & AP positivo & AP negativo & Total \\
\hline US positivo & 6 & 6 & 12 \\
US negativo & 2 & 1 & 3 \\
Total & 8 & 7 & 15 \\
\hline
\end{tabular}

Teste kappa: 0,11 - concordância muito fraca entre os métodos, p>0,05 (Fisher). Sensibilidade: $75 \%$; especificidade: $14 \%$; Valor preditivo positivo: $50 \%$; valor preditivo negativo: $33 \%$.

parede miometrial nas seqüências T2 de alta resolução (HASTE e TURBO FISP) (Figuras 3 e 4).

Nas seqüências contrastadas dinâmicas, obtidas em ecogradiente ponderadas em T1 após utilização de gadolíneo endovenoso, as fases arterial, venosa e tardia foram valorizadas com a identificação dos vasos invadindo o miométrio. O grau de extensão da invasão também foi avaliado nestas seqüências, demonstrados conforme as Figuras 1, 2, 3 e 4.

\section{Discussão}

A decídua endometrial normalmente serve como barreira à invasão trofoblástica. Uma vez que ocorre a invasão miometrial do vilo corial, essa placenta passa a se chamar placenta acreta. Placenta acreta corresponde a um espectro de anormalidades de implantação conhecidas como placenta acreta
Tabela 4 - Concordância entre a ressonância magnética (RM) e o resultado anátomo-patológico (AP).

\begin{tabular}{lccc}
\hline Teste & AP positivo & AP negativo & Total \\
\hline RM positiva & 8 & 1 & 9 \\
RM negativa & 0 & 6 & 6 \\
Total & 8 & 7 & 15
\end{tabular}

Teste kappa: 0,86 - houve concordância excelente entre os métodos, $p \leq 0,05$ (Fischer). Sensibilidade: $100 \%$; especificidade: $86 \%$; valor preditivo positivo: $89 \%$; valor preditivo negativo: $100 \%$

verdadeira, increta e percreta, graduadas de acordo com a profundidade de penetração do tecido placentário. A placenta acreta verdadeira é definida como aquela em que a aderência ao miométrio é secundária a uma ausência focal da decídua basal. Placenta increta significa que há uma invasão do vilo coriônico até o miométrio e a placenta percreta é aquela cujo vilo coriônico penetra até a serosa uterina. Estas anormalidades de implantação geralmente permanecem sem diagnóstico até o trabalho de parto e elevam o risco de morbimortalidade materna, principalmente quando ocorrem sangramentos incontroláveis durante o trabalho de parto ${ }^{8,9}$.

A decídua basal é derivada do endométrio e perdas focais da decídua ou doenças do endométrio podem repercutir em deficiência da decídua durante a gestação. Cirurgias uterinas prévias, incluindo cesáreas, curetagens, miomectomias e, mais recentemente, a embolização de miomas, têm sido relatadas como fatores de risco para essa condição ${ }^{10}$. 
O diagnóstico é muito dificil, no entanto, sinais ultra-sonográficos desta entidade têm sido bem estabelecidos nos últimos 20 anos. Em 1983, foi reportado o primeiro caso de placenta acreta como uma descontinuidade da zona hipoecóica retroplacentária, secundária à aderência placentária ${ }^{10}$.

A descontinuidade com afilamento da zona hipoecóica retroplacentária é considerada hoje como o indicador real de acretismo placentário na US. Em 1992, foi afirmado que a presença de numerosas lacunas vasculares é um critério adicional para o diagnóstico do acretismo. Avanços recentes na US com dopler colorido e power Doppler permitiram o estudo dos vasos e a mensuração da velocidade e fluxo do sangue placentário. Os padrões de pulsatilidade venosa associados à presença de lacunas venosas e ao fluxo venoso complexo retroplacentário são considerados critérios doplerfluxométricos para placenta acreta ${ }^{2,11,12}$.

Achados na RM de acretismo placentário, como o hipersinal placentário invadindo o miométrio, foram primeiramente descritos em $1992^{13}$.

A partir desse trabalho, outros começaram a ser desenvolvidos com o intuito de estabelecer o valor dos métodos de diagnóstico na avaliação do acretismo placentário. Em 1997, foi realizado um estudo com 18 casos comparando a US e a RM, incluindo seis casos de placenta acreta diagnosticados pela perda das características normais da zona hipoecóica retroplacentária, tais como o afilamento e a descontinuidade da serosa uterina. Placenta acreta foi aventada em cinco dos seis casos na seqüência turbo spin echo (TSE) ou "rapid acquisition with the relaxation enhancement" (RARE). Os autores concluíram que a RM não aumentava o diagnóstico de acretismo placentário, pois a escala de cinzas da US demonstrava os sinais satisfatoriamente, com boa sensibilidade e especificidade na maioria dos casos que tinham cicatriz de cesárea quando a placenta era localizada na parede anterior do útero ${ }^{3}$. Esses autores demonstraram também que a RM era a modalidade de escolha apenas nos casos de placentas corporais posteriores $^{3}$. Em nosso estudo, não utilizamos a seqüência TSE devido ao alto tempo de aquisição e ao grande número de artefatos, bem como ao elevado desconforto para a paciente gestante. Por isso, utilizamos as seqüências rápidas ponderadas em T2, como a HASTE e a TURBO FISP, que apresentam alta resolução de imagem e um tempo de aquisição curto (30 segundos); essas seqüências correspondem à seqüência RARE do trabalho citado anteriormente. Provavelmente, a dificuldade dos autores na definição da acuidade da RM foi devida à utilização de um protocolo com baixa resolução, alto tempo de aquisição (três minutos), elevado número de artefatos (movimentação fetal, movimentos respiratórios e peristálticos maternos) e ausência de fases com contraste. As fases dinâmicas permitem uma boa distinção da placenta e do miométrio; sua ausência certamente prejudicou os resultados destes autores.

A placenta é definida como um hipersinal homogêneo na cavidade uterina, e não é possivel diferenciar o vilo corial da decídua basal nas seqüências TSE ponderadas em $\mathrm{T} 2{ }^{3}$. Na escala de cinzas da US, porém, é possivel diagnosticar a descontinuidade do espaço retroplacentário e isso explica os resultados da autora citada anteriormente, os quais não correspondem aos achados da maioria dos trabalhos atuais da literatura.

Com intuito de avaliar a utilização do contraste no estudo da placenta, em 1997 foi desenvolvido um trabalho definindo o comportamento de realce da placenta na RM; assim, a placenta normal no terceiro trimestre exibe um realce lobular, que permite a diferenciação do vilo corial, ao contrário do segundo trimestre, quando a placenta apresenta um realce heterogêneo sem a definição de lóbulos ${ }^{14}$. Este autor realizou um estudo dinâmico depois de 45 segundos de injeção de contraste e não conseguiu diferenciar as regiões da placenta e do miométrio no segundo trimestre. Em nosso estudo, realizamos uma fase vascular precoce (arterial), com aproximadamente 15 segundos, e uma fase portal em que houve o realce lobular com 30 segundos. Isto permitiu a diferenciação das duas partes da placenta e a identificação da penetração de vasos coriais no miométrio. Essas fases permitiram uma associação praticamente sobreposta das peças cirúrgicas com as imagens da RM.

Com o mesmo intuito, em 2001, outro trabalho conseguiu distinguir as duas partes da placenta: os múltiplos focos lobulares contrastados e o realce ao redor dos lóbulos. O contraste dos lóbulos foi máximo aos 59 segundos. O realce precoce da face fetal da placenta permitiu a identificação do acretismo placentário, que depois foi confirmado no $\mathrm{AP}^{15}$.

Em nosso estudo, observamos que fases mais precoces e rápidas realizadas antes do realce do miométrio e dos lóbulos placentários, quando ainda existe pouco contraste na pelve, permitiram um diagnóstico mais fácil e fidedigno. Isso possibilitou estabelecer um protocolo bastante completo e com alta sensibilidade.

Neste trabalho, definimos dois critérios diagnósticos da placenta acreta na RM: o primeiro é o afilamento e a descontinuidade da decídua basal e da parede miometrial, bem caracterizadas nas seqüências rápidas ponderadas em T2 (TURBO FISP e HASTE); o segundo (na fase dinâmica) é o realce precoce de vasos tortuosos saindo da placenta 
fetal para a face materna e atingindo o miométrio. O primeiro sinal já havia sido demonstrado na maioria dos trabalhos ${ }^{1,2,15}$; já o segundo foi melhor delineado neste estudo com a utilização de fases mais precoces.

Em 2005, foi publicado um estudo multicêntrico com 300 casos com suspeita de acretismo, a maior série já estabelecida com exames de RM; porém, o contraste foi utilizado em apenas três casos e a realização de exames não seguiu um protocolo único, o que dificulta a definição precisa do papel da RM, com protocolos e técnicas de realização dos exames tão distintas. Este autor concluiu que a RM é fundamental para definição da topografia e da área de invasão placentária e que os achados da RM modificaram de maneira significativa a tática e a técnica cirúrgica, reduzindo a morbimortalidade e aumentando significantemente o número de cirurgias conservadoras ${ }^{16}$. Em nosso estudo, o achado mais relevante foi a mudança na tática cirúrgica e a melhor acurácia da RM em relação ao US.

Em conclusão, parece realmente existir uma boa correlação anátomo-patológica da RM na avaliação do acretismo placentário. Existem sinais específicos que devem ser conhecidos pelo radiologista na avaliação dessa anormalidade.

As seqüências rápidas ponderadas em T2 (TURBO FISP e HASTE) apresentam uma ótima resolução de imagem por serem rápidas e de alta resolução espacial e permitem caracterizar a parede miometrial. Em 2004, um estudo demonstrou a vantagem das imagens rápidas (TURBO FISP e HASTE), melhorando significantemente a avaliação anatômica da interface útero-placentária, e que essas duas seqüências se complementam ${ }^{17}$. No nosso trabalho também foram utilizadas as mesmas seqüências. Nesse trabalho, o contraste foi útil na avaliação dos vasos e realce, demonstrando um excelente contraste entre a placenta e o miométrio. Os autores concluíram que a RM dinâmica é uma técnica promissora para o diagnóstico pré-natal do acretismo placentário, especialmente em casos de placentas corporais posteriores. Em um artigo de revisão recentemente publicado, foi demonstrado que o uso de compostos com gadolíneo como contraste melhora a caracterização do acretismo placentário e pode melhorar a sensibilidade e a espeficidade do acretismo placentário ${ }^{18}$. Esses achados foram muito semelhantes aos nossos resultados.

O diagnóstico pré-natal da placenta acreta tem melhorado recentemente, com a combinação das técnicas de diagnóstico. Isso permitirá um real benefício para as populações de alto risco, com a redução da mortalidade, já que a prevalência do acretismo tem aumentado significantemente nos últimos 50 anos.

\section{Agradecimentos}

Às pacientes gestantes e seus recém-nascidos que contribuiram para este estudo e ao Departamento de Ginecologia e ao Departamento de Obstetricia da UNIFESP.

\section{Referências}

1. Gielchinsky Y, Rojansky N, Fasouliotis SJ, Ezra Y. Placenta accreta- summary of 10 years: a survey of 310 cases. Placenta. 2002;23(2-3):210-4

2. Finberg HJ, Williams JW. Placenta accreta: prospective sonographic diagnosis in patients with placenta previa and prior cesarean section. J Ultrasound Med. 1992;11(7):333-43.

3. Levine D, Hulka CA, Ludmir J, Li W, Edelman RR. Placenta accreta: evaluation with color Doppler US, power Doppler US and MR imaging. Radiology. 1997;205(3):773-6.

4. Lam G, Kuller J, McMahon M. Use of magnetic resonance imaging and ultrasound in the antenatal diagnosis of placenta accreta. J Soc Gynecol Investig. 2002;9(1):1-3.

5. Madjian C, Adam R, Pelosi M, Pelosi M 3rd, Rudelli RD, Maldjian J. MRI appearance of placenta percreta and placenta accreta. Magn Reson Imaging. 1999;17(7):965-71.

6. Torloni MR, Moron AF, Camano L. Placenta prévia: fatores de risco para o acretismo. Rev Bras Ginecol Obstet. 2001;23(7):417-22.

7. Paublo MM, Bustos Vidal JC, Ramirez HP, Abud DM, Rojas GC, Avilla P. Diagnóstico prenatal de placenta acreta por ultrasonido Doppler. Rev Chil Ultrason. 2000;3(3):80-2.

8. Kapernick OS. Postpartum hemorrhage, the anormal pierperium. In: Pernoll ML, editor. Current obstetric and gynecologic diagnosis \& treatment. 7th ed. East Norwalk: Appleton \& Lange; 1984. p. 568-88.

9. Pritchard JA, Mac Donald PC, Gant NF. Williams obstetrics. Norwalk: Appleton-Century-Crofts; 1985.

10. Tabsh KM, Brinkman CR 3rd, King W. Ultrasound diagnosis of placenta increta. J Clin Ultrasound. 1982;10(6):288-90.

11. Chou MM, Ho ES. Prenatal diagnosis of placenta previa accreta with power amplitude ultrasonic angiography. Am J Obstetric Gynecol. 1997;177(6):1523-5.

12. Lerner JP, Deane S, Timor-Tritsch IE. Characterization of placenta accreta using transvaginal sonography, and color Doppler imaging. Ultrasound Obstetr Gynecol. 1995;5(3):198-201.

13. Thorp JM Jr, Councell RB, Sandridge DA, Wiest HH. Antepartum diagnosis of placenta previa percreta

Rev Bras Ginecol Obstet. 2006; 28(12): 700-7. 
by magnetic resonance imaging Obstet Gynecol. 1992;80(3 Pt 2):506-8.

14. Marcos HB, Semelka RC, Worawattanakul S. Normal placenta: gadolinium-enhanced dynamic MR imaging. Radiology. 1997;205(2):493-6.

15. Tanaka YO, Sohda S, Shigemitsu S, Niitsu M, Itai Y. High temporal resolution dynamic contrast MRI in a high risk group for placenta accreta. Magn Reson Imaging. 2001;19(5):635-42.

16. Palacios Jaraquemada JM, Bruno CH. Magnetic resonance imaging in 300 cases of placenta accreta: surgical correlation of new findings. Acta Obstet Gynecol Scand. 2005;84(8):716-24.

17. Kim JA, Narra VR. Magnetic resonance imaging with true fast imaging with steady-state precession and half-Fourier acquisition single-shot turbo spin-echo sequences in cases of suspected placenta accreta. Acta Radiol. 2004;45(6):692-8.

18. Mauzoni C, Gorincour G, Juhan V, Bretelle F. Placenta accreta: a review of current advances in prenatal diagnosis. Placenta. 2006 Sep 4; [Epub ahead of print]. 